\title{
Honorarzuwachs 2011: Das sieht sehr nach Roulette aus
}

— Der Erweiterte Bewertungsausschuss hat am 5. Oktober 2010 einen Grundsatzbeschluss zur sog. asymmetrischen Verteilung eines bundesweiten Gesamtvolumens zur Anhebung der ambulanten Honorare in Höhe von 500 Mio. Euro gefasst. Beschlossen wurde ein von der KBV entwickeltes sog. Modell 3d, das von einer linearen Anhebung in Höhe von $0,75 \%$ der morbiditätsbedingten Gesamtvergütungen aller KVen ausgeht.

Die so angehobene morbiditätsbedingte Gesamtvergütung je Versicherten bzw. der linear gesteigerte morbiditätsbedingte Behandlungsbedarf je Versicherten stellt die Grundlage der Einsortierung der KVen in drei Klassen mit unterschiedlichen maximalen prozentualen $\mathrm{Zu}$ wachsgruppen dar.
In Klasse I kommt ein Anstieg von 5\% zustande, wenn 9235 Punkte (324 Euro) unterschritten werden. In Klasse II beträgt der Anstieg 4\%, wenn sich der Leistungsbedarf zwischen 9235 und 9655 Punkten (338 Euro) bewegt, und in Klasse III erfolgt ein Anstieg um o,86\%, wenn 9655 Punkte überschritten werden. Es gibt allerdings die mündliche Zusage des Bundesministeriums für Gesundheit, dass die lineare Anhebung auf 1,5\% verdoppelt wird. In diesem Fall ändert sich die Verteilung in den 3 Klassen nochmals und es kommt zu dem in der Tabelle dargestellten Ergebnis.

\section{MMW Kommentar}

Die nunmehr im Erweiterten Bewertungsausschuss beschlossene regionale Zuteilung der für 2011 für den ambulanten Be- reich zur Verfügung gestellten Geldmenge orientiert sich ausschließlich am sogennannten Leistungsbedarf in den KVen in der Vergangenheit. Regionale Besonderheiten, wie z.B. das ambulante Leistungsspektrum in einer $K V$, wurden dabei nicht berücksichtigt. Das führt zu der kuriosen Situation, dass nunmehr KVen eine höhere Mittelzuteilung erhalten, obgleich sie über Jahre hinweg zuvor einen geringen Leistungsbedarf der Bevölkerung dokumentiert haben und umgekehrt. Die Folge dieser Mittel(um)verteilung wird deshalb zwangsläufig eine Art Normierung der ambulanten Versorgung sein müssen mit dem Ziel, möglichst viele Leistungen in andere Sektoren, wie z.B. den stationären Bereich, umzulenken.

\begin{tabular}{|c|c|c|c|c|c|c|c|c|c|}
\hline \multirow[t]{2}{*}{$\begin{array}{l}\text { Kassenärztliche } \\
\text { Vereinigung }\end{array}$} & \multirow{2}{*}{$\begin{array}{l}\text { MGV je } \\
\text { V. } 2010 \\
\text { Euro }\end{array}$} & \multirow{2}{*}{$\begin{array}{c}\text { BB je } \\
\text { V. } 2010 \\
\text { Punkte }\end{array}$} & \multirow{2}{*}{$\begin{array}{c}\text { MGV je V. } 2011 \\
(\text { VR = 1,5\%) } \\
\text { Euro }\end{array}$} & \multirow{2}{*}{$\begin{array}{c}\text { BB je } \\
\text { V. } 2011 \\
\text { Punkte }\end{array}$} & \multirow{2}{*}{$\begin{array}{c}\text { MGV je V. } 2011 \\
\text { Asym. } \\
\text { Euro }\end{array}$} & \multicolumn{2}{|c|}{$\begin{array}{l}\text { Differenz aus } \\
\text { Asymmetrie }\end{array}$} & \multicolumn{2}{|c|}{$\begin{array}{l}\text { Differenz } 2011 \\
\text { zu MGV } 2010\end{array}$} \\
\hline & & & & & & Mio. Euro & Prozent & Mio. Euro & Prozent \\
\hline 1 & 2 & 3 & 4 & 5 & 6 & 7 & 8 & 9 & 10 \\
\hline Schleswig-Holstein & 320 & 9143 & 325 & 9534 & 334 & 27 & $3,46 \%$ & 38 & $4,98 \%$ \\
\hline Hamburg & 369 & 10535 & 375 & 10614 & 372 & 0 & $0,00 \%$ & 8 & $1,50 \%$ \\
\hline Bremen & 361 & 10288 & 366 & 10466 & 367 & 2 & $0,97 \%$ & 5 & $2,47 \%$ \\
\hline Niedersachsen & 346 & 9869 & 351 & 10041 & 352 & 25 & $1,05 \%$ & 60 & $2,56 \%$ \\
\hline Westfalen-Lippe & 305 & 8715 & 310 & 9088 & 319 & 78 & $3,46 \%$ & 111 & $4,98 \%$ \\
\hline Nordheim & 315 & 8995 & 320 & 9380 & 329 & 88 & $3,46 \%$ & 126 & $4,98 \%$ \\
\hline Hessen & 342 & 9768 & 347 & 9939 & 348 & 18 & $1,05 \%$ & 44 & $2,56 \%$ \\
\hline Rheinland-Pfalz & 325 & 9276 & 330 & 9603 & 337 & 30 & $2,72 \%$ & 47 & $4.24 \%$ \\
\hline Baden-Württemberg & 330 & 9426 & 335 & 9758 & 342 & 80 & $2,72 \%$ & 124 & $4,24 \%$ \\
\hline Bayern & 359 & 10248 & 365 & 10427 & 365 & 40 & $1,05 \%$ & 96 & $2,56 \%$ \\
\hline Berlin & 381 & 10866 & 387 & 10947 & 384 & 0 & $0,00 \%$ & 16 & $1,50 \%$ \\
\hline Saaland & 359 & 10251 & 365 & 10430 & 366 & 3 & $1,05 \%$ & 8 & $2,56 \%$ \\
\hline Mecklenburg-Vorp. & 366 & 10451 & 372 & 10529 & 369 & 0 & $0,00 \%$ & 8 & $1,50 \%$ \\
\hline Brandenburg & 323 & 9216 & 328 & 9610 & 337 & 25 & $3,46 \%$ & 36 & $4,98 \%$ \\
\hline Sachsen-Anhalt & 310 & 8833 & 314 & 9211 & 323 & 23 & $3,46 \%$ & 33 & $4,98 \%$ \\
\hline Thüringen & 326 & 9305 & 331 & 9632 & 338 & 18 & $2,72 \%$ & 28 & $4,24 \%$ \\
\hline Sachsen & 323 & 9225 & 328 & 9620 & 337 & 43 & $3,46 \%$ & 61 & $4,98 \%$ \\
\hline Gesamt & 335 & 9556 & 340 & 9833 & 345 & 500 & $2,13 \%$ & 850 & $3,64 \%$ \\
\hline Maximum & 381 & & 387 & & 384 & & & & \\
\hline Minimum & 305 & & 310 & & 319 & & & & \\
\hline Spannbreite & 74 & & 77 & & 65 & & & & \\
\hline
\end{tabular}

So könnte die Honorarverteilung 2011 aussehen. Sicher ist das aber noch nicht und zweckmäßig schon gar nicht. 